\title{
Physics at FCC-ee
}

\section{Tadeusz Lesiak*}

The H.Niewodniczański Institute of Nuclear Physics

Polish Academy of Sciences, Kraków

E-mail: Tadeusz.Lesiak@ifj.edu.pl

The potential of physics studies at the Future Circular Collider with $e^{+} e^{-}$beams (FCC-ee) is briefly discussed. Special attention is paid to the measurements in the sector of quantum chromodynamics

The European Physical Society Conference on High Energy Physics (EPS-HEP 2019)

10-17 July, 2019

Ghent, Belgium

* Speaker.

${ }^{\dagger}$ On behalf of the FCC Design Study Group 


\section{Introduction}

The Future Circular Collider (FCC) design study [1] assumes the construction and exploitation of a $\sim 100 \mathrm{~km}$ circular tunnel infrastructure in the Geneva area. The Future Circular Collider with $e^{+} e^{-}$beams (FCC-ee) is proposed as the first phase of the project in the perspective of the successful physics program undertaken at LEP accelerator [2] and in view of the Higgs boson discovery [3] in 2012. In turn, the FCC-ee is envisioned to be replaced by the $100 \mathrm{TeV}$ protonproton collider FCC-hh. At the same time, the collisions of $60 \mathrm{GeV}$ electrons from an energy recovery linac with $50 \mathrm{TeV}$ protons, can be realized (FCC-eh). The efforts of the FCC design study have been collected in the Conceptual Design Report [4], issued in March 2019, and submitted as input to the European Particle Physics Strategy Update 2018-2020 [5]. The relevant, general information about the FCC-ee project can be found in $[6,7,8]$.

The FCC-ee collider will profit from recent innovations, implemented at $b$-factories, like the nanobeam scheme, continuous injection and crab-crossing optics. The collider would collect data at four center-of-mass $(\mathrm{CM})$ energies $(\sqrt{s})$, corresponding to the $Z$ pole and the $W W, H Z$ and $t \bar{t}$ thresholds. Its most relevant parameters [4] are presented in Table 1 and compared with LEP phase-2 (LEP2) collider [2]. At least two general-purpose spectrometers will be operating at the interaction points. Such detectors should be hermetic, lightweight in terms of the material content and provide very accurate tracking and fine-granularity calorimetry.

In the following chapters, the Higgs, $\mathrm{Z}$ and $\mathrm{W}$ bosons and top quark studies at the FCC-ee will be briefly reviewed. Finally the potential of QCD measurements will be discussed.

\begin{tabular}{l|c|cccc}
\multicolumn{1}{c|}{ Parameter } & LEP2 & \multicolumn{4}{c}{ FCC-ee } \\
\hline \hline$E_{b}[\mathrm{GeV}]$ & 104 & 45.6 & 80 & 120 & $175-182.5$ \\
$I[\mathrm{~mA}]$ & 4 & 1390 & 147 & 29 & 5.4 \\
$\#$ of bunches/beam & 4 & 16640 & 2000 & 393 & 48 \\
$\beta_{x}^{*}[\mathrm{~m}]$ & 1.5 & 0.15 & 0.2 & 0.3 & 1.0 \\
$\beta_{y}^{*}[\mathrm{~mm}]$ & 50 & 0.8 & 1 & 1 & 1.6 \\
$\varepsilon_{x}[\mathrm{~nm}]$ & $30-50$ & 0.27 & 0.28 & 0.63 & 1.46 \\
$\varepsilon_{y}[\mathrm{pm}]$ & 250 & 1 & 1.7 & 1.3 & 2.9 \\
$P_{\mathrm{SR}}[\mathrm{MW}]$ & 22 & 100 & 100 & 100 & 100 \\
\hline$L\left[10^{34} \mathrm{~cm}^{-2} \mathrm{~s}^{-1} / \mathrm{IP}\right]$ & 0.012 & 230 & 28 & 8.5 & 1.55 \\
$L_{\text {tot }}\left[\mathrm{ab}{ }^{-1}\right]$ & $8 \times 10^{-4}$ & 150 & 10 & 5 & 1.7 \\
\hline Statistics & & $\sim 5 \times 10^{12} Z$ & $\sim 10^{8} W W$ & $\sim 10^{6} \mathrm{ZH}$ & $\sim 10^{6} t \bar{t}$ \\
$(2$ expts $)$ & & $(4$ years $)$ & $(2$ years $)$ & $(3$ years $)$ & $(5$ years $)$
\end{tabular}

Table 1: Selected parameters of the FCC-ee accelerator. The characteristics of LEP2 collider have been supplemented for comparison. The following parameters are presented: beam energy $\left(E_{b}\right)$, beam current $(I)$, number of bunches, horizontal (vertical) beta function at the interaction point (IP) $\beta_{x}^{*}\left(\beta_{y}^{*}\right)$, horizontal (vertical) emittance $\left(\varepsilon_{x}\left(\varepsilon_{y}\right)\right)$, synchrotron radiation power $\left(P_{\mathrm{SR}}\right)$, luminosity $(L)$ and expected statistics of relevant events (from [4]). 


\section{Higgs boson physics at the FCC-ee}

FCC-ee will measure Higgs boson production inclusively from its presence as a recoil to the $\mathrm{Z}$ in the process $e^{+} e^{-} \rightarrow Z H$ (cf. Fig. 1 from [4]). The resulting expected precision of the Higgs mass amounts to $10 \mathrm{MeV}$. The $H Z$ cross-section $\left(\sigma_{H Z}\right)$ is proportional to the square of the Higgs boson coupling to the $\mathrm{Z}, g_{H Z Z}$. The recoil method allows as well for the determination of the Higgs boson width $\left(\Gamma_{H}\right)$ by counting the number of $H Z$ events in which the Higgs decays into a pair of $Z$ bosons $\left(\sigma_{H Z} \times \Gamma(H \rightarrow Z Z) / \Gamma_{H} \propto g_{H Z Z}^{4} / \Gamma_{H}\right)$. In turn, the numbers of events with Higgs boson exclusive decays into $X X=b \bar{b}, c \bar{c}, \tau^{+} \tau^{-}, \mu^{+} \mu^{-}, \gamma \gamma, g g, W^{+} W^{-}$, and invisible Higgs boson decays, allow to measure the respective Higgs couplings, $g_{H X X}$, with the accuracy below $1 \%$ [4].
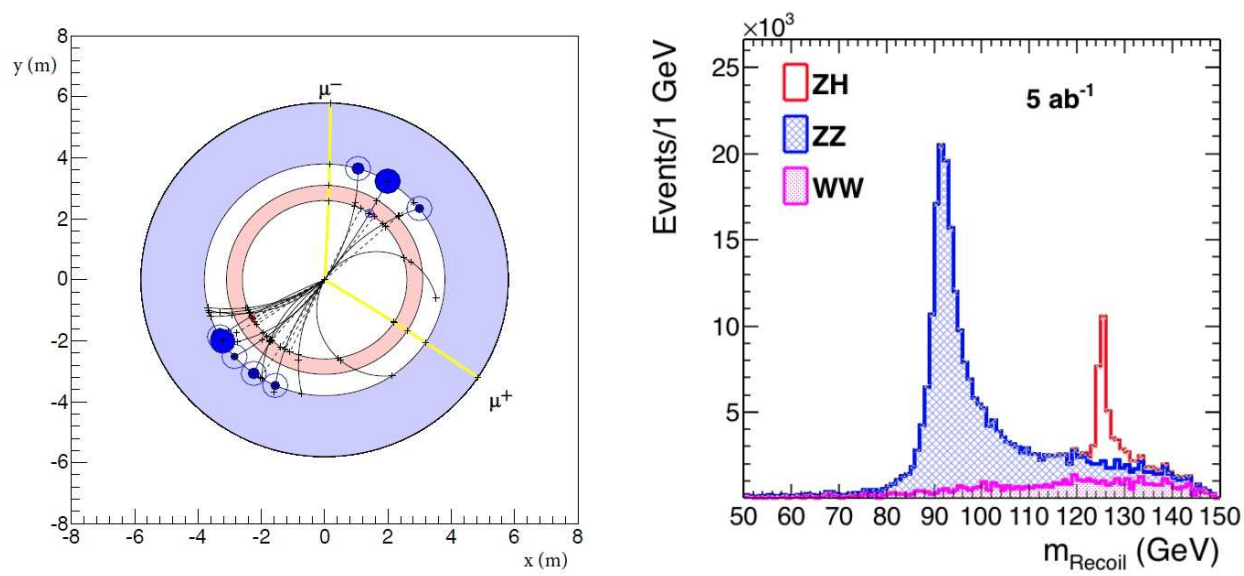

Figure 1: Left: A schematic view, transverse to the detector axis, of an $e^{+} e^{-} \rightarrow H Z$ event with $Z \rightarrow$ $\mu^{+} \mu^{-}$and with the Higgs boson decaying hadronically. The two muons from the $Z$ decay are indicated. Right: Distribution of the mass recoiling against the muon pair, determined from the total energy-momentum conservation, with an integrated luminosity of $5 \mathrm{ab}^{-1}$. The peak around $125 \mathrm{GeV}$ (in red) consists of $\mathrm{HZ}$ events. The rest od the distribution (in blue and pink) originate from $Z Z$ and $W W$ production.

\section{Electroweak precision measurements at the $Z$ pole}

Precision electroweak measurements are at the center of the FCC-ee physics program, as they can play a key role in establishing the existence of new physics together with elucidating its theoretical interpretation. The expected FCC-ee data statistics of $5 \times 10^{12} \mathrm{Z}$ events corresponds to the increase by five orders of magnitude to compare with data sample collected at LEP1. This implies, that the overall precision of electroweak observables at the FCC-ee will be generally limited by systematic uncertainties. Thus, updated, more accurate theoretical calculations of electroweak observables are to be undertaken. Special attention should be as well paid to the accurate CM energy calibration, realized via resonant depolarisation. The corresponding systematic uncertainty of $\leq 100 \mathrm{keV}$ is expected on both the mass and width of the $Z$ boson (to compare with $\Delta M_{Z}=2.1 \mathrm{MeV}$ and $\Delta \Gamma_{Z}=2.3 \mathrm{MeV}$ at LEP1). The experimental precision of the selected electroweak observables, expected at FCC-ee, is presented in Table 2. In particular, an improved determination of the electromagnetic coupling constant at the $Z$ mass $\left(\alpha_{\mathrm{QED}}\left(m_{Z}\right)\right)$ [9] can be obtained using direct method 
based on the forward-backward muon asymmetry measurement at two optimal working points $\left(\sqrt{s_{-}}=87.9 \mathrm{GeV}\right.$ and $\left.\sqrt{s_{+}}=94.3 \mathrm{GeV}\right)$.

\begin{tabular}{l|c|c|c|c} 
Observable & $\begin{array}{c}\text { present value } \\
\pm \text { error }\end{array}$ & $\begin{array}{c}\text { FCC-ee } \\
\text { Stat. }\end{array}$ & $\begin{array}{c}\text { FCC-ee } \\
\text { Syst. }\end{array}$ & $\begin{array}{c}\text { Improvement } \\
\text { factor }\end{array}$ \\
\hline$m_{Z}\left[\mathrm{keV} / \mathrm{c}^{2}\right]$ & $91186700 \pm 2200$ & 5 & 100 & 22 \\
$\Gamma_{Z}[\mathrm{keV}]$ & $2495200 \pm 2300$ & 8 & 100 & 23 \\
$R_{l}^{Z}\left[\times 10^{3}\right]$ & $20767 \pm 25$ & 0.06 & $0.2-1$ & $125-25$ \\
$\alpha_{S}\left(m_{Z}\right)\left[\times 10^{4}\right]$ & $1196 \pm 30$ & 0.1 & $0.4-1.6$ & $75-19$ \\
$R_{b}\left[\times 10^{6}\right]$ & $216290 \pm 660$ & 0.3 & $<60$ & 11 \\
$N_{v}\left[\times 10^{3}\right]$ & $2991 \pm 7$ & 0.005 & 1 & 7 \\
$\sin ^{2} \theta_{W}^{\text {eff }}\left[\times 10^{6}\right]$ & $231480 \pm 160$ & 3 & $2-5$ & $44-28$ \\
$1 / \alpha_{\mathrm{QED}}\left(m_{Z}\right)\left[\times 10^{3}\right]$ & $128952 \pm 14$ & 4 & small & 3.5 \\
$A_{\mathrm{FB}, 0}^{b}\left[\times 10^{4}\right]$ & $992 \pm 16$ & 0.02 & $1-3$ & $16-5$ \\
$A_{\mathrm{FB}}^{\mathrm{pol}, \tau}\left[\times 10^{4}\right]$ & $1498 \pm 49$ & 0.15 & $<2$ & 25
\end{tabular}

Table 2: Measurements of selected electroweak observables, compared with the present accuracy.

\section{Top quark and $W$ boson properties at the FCC-ee}

FCC-ee aims at the collection of enormous samples at the thresholds for the production of $W W$ pairs $\left(\sim 10^{8} \mathrm{WW}\right.$ events) and $t \bar{t}$ pairs $\left(\sim 10^{6} t \bar{t}\right.$ events). Assmuning two, equally shared, working points at $\sqrt{s}=157.5 \mathrm{GeV}$ and $\sqrt{s}=162.5 \mathrm{GeV}$, a simultaneous fit of the $W$ mass and width to the $e^{+} e^{-} \rightarrow W^{+} W^{-}$cross-sections measurements, is expected to yield an accuracy of $0.5 \mathrm{MeV}$ on $m_{W}$ and $1.2 \mathrm{MeV}$ on $\Gamma_{W}$ [4]. For the top quark, the optimal strategy is a multipoint scan in the CM energy range (340-345) GeV, leading to statistical precisions of $\pm 17 \mathrm{MeV}( \pm 45 \mathrm{MeV})$ for the top-quark mass (width), respectively [4].

\section{QCD studies at the FCC-ee}

Studies of strong interactions in an extremely clean environment of high-luminosity $e^{+} e^{-}$ collisions offer several significant advantages, as proved by a very successful QCD program, undertaken at LEP [10]. First of all, one deals with a fully-controlled QED initial state with known kinematics. Moreover, QCD phenomena manifest itself only in the final state and the resulting difficulties due to beam remnants and initial state parton distribution functions are absent. For FCC-ee at the $Z$ peak, one expects data samples of hadronic events $10^{5}$ larger than at LEP, The respective gain in statistics at the $W^{+} W^{-}$threshold amounts to $10^{3}$. Moreover, the hadronic samples from Higgs boson ( $10^{5}$ events) and top-quark will be available for the first time in $e^{+} e^{-}$collisions. In view of these assets, the three main objectives of QCD program at FCC-ee are:

1. determination of the strong interaction coupling constant $\alpha_{s}$ with the permille accuracy, to compare with the current precision of $1 \%$, 
2. high-precision studies of perturbative parton radiation encompassing high-order leading corrections and logarithmic resummations for event angularities (shapes), jet multiplicities, jet substructure, quark/gluon/heavy-quark discrimination, and $q, g, c, b$ parton-to-hadron fragmentation functions,

3. high-precision non-perturbative QCD studies including color reconnection, parton hadronisation, final-state multiparticle correlations, very rare hadron production and decays, to name but a few.

The extraction of $\alpha_{s}$ will be based on huge samples of hadronic $\tau, W$ and $Z$ events studied with $\mathrm{N}^{3} \mathrm{LO}$ theoretical precision. For $\tau$ and $W$ the relevant quantities are the ratios of hadronic to leptonic widths. The expected accuracies are $<1 \%$ from $\tau$, and $\sim 0.3 \%$ from $W$ decays. At the $Z$ peak, the $\alpha_{s}$ coupling constant can be determined from studies of event angularities (shapes), $\mathrm{N}$-jet cross sections and jet rates, and from three hadronic observables: $\Gamma_{Z}, \sigma_{0}^{\text {had }}=12 \pi / m_{Z} \cdot \Gamma_{e} \Gamma_{\text {had }} / \Gamma_{Z}^{2}$ and $R_{l}^{0}=\Gamma_{\text {had }} / \Gamma_{l}$. The expected accuracy of the $\alpha_{s}$ extraction from $Z$ decays amounts to $\sim 0.2 \%$.

It is estimated [4], that jet rates will be determined by FCC-ee at the $Z$ pole with $10^{-6}$ precision for: 4-jet events up to the scale $k_{T} \sim 30 \mathrm{GeV}$ (corresponding to $|\ln (y)| \sim 2$, for jet resolution parameter $\left.y=k_{T}^{2} / s\right), 5$-jet events at $k_{T} \sim 20 \mathrm{GeV}(|\ln (y)| \sim 3)$, 6-jet events at $k_{T} \sim 12 \mathrm{GeV}$ $(|\ln (y)| \sim 4)$ and 7-jet events at $k_{T} \sim 7.5 \mathrm{GeV}(|\ln (y)| \sim 5)$.

Event angularities (shapes) are affected by logarithmic enhancements, which are resummed nowadays up to $\mathrm{N}^{3} \mathrm{LO}$, and by hadronisation corrections, estimated with Monte Carlo generators. Consequently, studies of these observables yields insight into resummation and hadronisation effects. In case of the FCC-ee, analyses of data collected at different CM energies are expected to diminish non-perturbative uncertainties at the $Z$ peak from $9 \%$ to $2 \%$.

The FCC-ee studies of event angularities, based on $Z \rightarrow q \bar{q}$ and $H \rightarrow g g$ samples, can also provide an important insight into the patterns of quark/gluon radiation and, correspondingly, into the discrimination between quark and gluon jets. In particular, there is currently a relatively good agreement between different Monte Carlo generators in the description of event angularities for quark jets. At the same time, however, the predictions of Monte Carlo generators for the gluon jets, vary significantly. Thus, FCC-ee can bring here crucial improvement in the quality of quarkqluon jet discriminators and in the description of gluon jets. Special attention will be also paid to ameliorations in the performance of taggers for $b$ and $c$ quark jets.

Studies of color reconnection (CR) effects, corresponding to the color flow between color singlets parton systems of different origin, are among the most important issues of non-perturbative QCD. The CR effects are best studied in $e^{+} e^{-}$annihilation in the process $e^{+} e^{-} \rightarrow q_{1} \bar{q}_{2} q_{3} \bar{q}_{4}$ with the formation of color singlets $\left(q_{1} \bar{q}_{2}\right)$ and $\left(q_{3} \bar{q}_{4}\right)$ or, alternatively, $\left(q_{1} \bar{q}_{4}\right)$ and $\left(q_{3} \bar{q}_{2}\right)$. The LEP results excluded the no-CR null hypothesis at $99.5 \%$ CL [12], but data were too scarce to provide any quantitative studies. The $10^{3}$ gain in statistics at FCC-ee with the associated precision of the $W$ mass determination at the level of $1 \mathrm{MeV}$, will allow to evaluate the presumable shift in the $m_{W}$ measured in hadronic events to compare with the semileptonic $W W$ pairs which are unaffected by CR effects. Moreover, such comparison can be performed in three different CM energies. As shown in Table 3, the mass shifts expected in different CR models differ significantly between the models together with exhibiting variations as a function of $\sqrt{s}$. 


\begin{tabular}{|c|c|c|c|c|c|c|c|}
\hline$\sqrt{s}$ & \multicolumn{7}{|c|}{$<\delta \bar{m}_{W}>[\mathrm{MeV}]$} \\
\cline { 2 - 8 }$[\mathrm{GeV}]$ & I & II & II' & GM-I & GM-II & GM-III & CS \\
\hline 170 & +18 & -14 & -6 & -41 & +49 & +2 & +7 \\
240 & +95 & +29 & +25 & -74 & +400 & +104 & +9 \\
350 & +72 & +18 & +16 & -50 & +369 & +60 & +4 \\
\hline
\end{tabular}

Table 3: Reconstructed average $W$ mass shift predicted by different CR models in PYTHIA 8, relative to the no-CR baseline, in $e^{+} e^{-} \rightarrow W^{+} W^{-} \rightarrow q_{1} \bar{q}_{2} q_{3} \bar{q}_{4}$ at three FCC-ee CM energies [13].

\section{Summary and acknowledgements}

The FCC-ee collider aims at ambitious physicis program which would qualitatively improve the experimental precision of practically all Standard Model observables. In particular a substantial progress is expected in the QCD studies.

I would like to thank Alain Blondel, David d'Enterria, Markus Klute and Peter Skands for their help in preparing the talk. This research was supported in part by grant of Narodowe Centrum Nauki 2016/23/B/ST2/03927.

\section{References}

[1] http://fcc.web.cern.ch

[2] A.Straessner, "Electroweak Physics at LEP and LHC", Springer, 2010.

[3] A. Nisati and V. Sharma, "Discovery of the Higgs Boson", World Scientific, 2017.

[4] A. Abada et al., Eur. Phys. J. C79 (2019) 474, http://fcc-cdr.web.cern.ch

[5] https://indico.cern.ch/event/765096/contributions/3298137/

[6] "CERN thinks bigger", CERN Courier magazine, June 2018, https: cerncourier.com/cern-thinks-bigger/

[7] A. Blondel et al., FCC-ee: Your Questions Answered, [hep-ex/1906.02693].

[8] M. Benedikt and F. Zimmermann, The physics and technology of the Future Circular Collider, Nature Rev. Phys. 1 (2019), 238.

[9] P. Janot, Direct measurement of $\alpha_{\mathrm{QED}}\left(m_{Z}^{2}\right)$ at the FCC-ee, JHEP 02 (2016) 053.

[10] S. Bethke, "QCD studies at LEP", Phys. Rept. 403-404 (2004), 203.

[11] A.J. Larkoski, J. Thaler and W.J. Waalewijn, "Gaining (Mutual) Information about Quark/Gluon Discrimination", JHEP 11 (2014), 129.

[12] The LEP Electroweak Working Group and the ALEPH,DELPHI, L3, OPAL Collaborations, "Electroweak Measurements in Electron-Positron Collisions at W-Boson Pair Energies at LEP", Phys. Rept. 532 (2013), 119.

[13] J.R. Christiansen and, T. Sjöstrand, "Color reconnection at future $e^{+} e^{-}$colliders", Eur. Phys. J. C75 no. 9 (2015), 441. 